\title{
Interfacial activity of patchy worm-like micelles $\dagger$
}

Cite this: Soft Matter, 2013, 9, 11173

\author{
Joachim Schmelz, $\$^{\mathrm{a}}$ Daniela Pirner, $\neq^{\mathrm{ab}}$ Marina Krekhova, ${ }^{\mathrm{a}}$ Thomas M. Ruhland ${ }^{\mathrm{a}}$ \\ and Holger Schmalz ${ }^{* a}$
}

Received 12th July 2013

Accepted 14th October 2013

DOI: $10.1039 / c 3 s m 51914 g$

www.rsc.org/softmatter

The interfacial activity of self-assembled worm-like crystalline-core micelles (wCCMs) with a patchy polystyrene/poly(methyl methacrylate) (PS/PMMA) corona and a semi-crystalline polyethylene (PE) core is studied at the toluene-water interface. Strikingly, the interfacial activity is comparable to that of Janus cylinders with well-separated PS/PMMA hemishells and comparable length. From this finding an adaptation of the corona chains of the patchy wCCMs to the toluene-water interface is proposed.

Surface compartmentalization in nanoparticles leads to a variety of unique fields of application, e.g. the self-assembly into hierarchical superstructures or the selective incorporation of multiple nanoparticles or dyes into defined spatial confinement. ${ }^{1,2}$ Another fascinating attribute of surface-compartmentalized nanostructures is their outstanding surface activity. The combination of the "Pickering effect"3-5 valid for particles at interfaces with the amphiphilicity of classical surfactants makes them ideal candidates for a new generation of superior surfactants or to produce functional assemblies. ${ }^{6}$

Research on surface-compartmentalized nanostructures until now has mainly focused on Janus particles that, named after the two-faced Roman god Janus, exhibit exactly two opposing hemishells of different chemistry and/or polarity. ${ }^{7,8}$ Dense, solid Janus colloids with sizes ranging in the micrometer region and slightly below have been synthesized using various techniques during the last decade, mainly resulting in spherical geometries. ${ }^{\mathbf{9 - 1 2}}$ Stepping down further in size, block terpolymer based spherical, cylindrical, ribbon-like and disc-like Janus micelles with crosslinked cores were prepared via a template-assisted approach. ${ }^{13-16}$

${ }^{a}$ Macromolecular Chemistry II, University of Bayreuth, 95440 Bayreuth, Germany. E-mail: holger.schmalz@uni-bayreuth.de

${ }^{b}$ Physical Chemistry I, University of Bayreuth, 95440 Bayreuth, Germany

$\dagger$ Electronic supplementary information (ESI) available: Experimental details, selected TEM micrographs of wCCMs, interfacial tension isotherms of the pristine toluene-water interface and for SEM1 wCCMs at the dioxane-PFO interface. See DOI: $10.1039 / \mathrm{c} 3 \mathrm{sm} 51914 \mathrm{~g}$

\$ J.S. and D.P. contributed equally to this work.
Triggered by theoretical studies predicting an up to 3-fold increase in the surface activity of spherical Janus structures with respect to homogeneous particles, ${ }^{17}$ experimental efforts to explore the potential of Janus particles as surfactants were undertaken. Böker and Krausch et al. observed a reduction of the oil-water interfacial tension for iron-oxide/gold Janus particles that was significantly higher than that observed for the respective uniform particles. ${ }^{18}$ Furthermore, Janus particles of varying geometry (spherical, cylindrical, and disc-like) prepared by the template-assisted approach were shown to exhibit superior surface activity compared to the unimolecularly dissolved triblock terpolymer precursors used for their synthesis. ${ }^{\mathbf{1 6 , 1 9 , 2 0}}$ For Janus cylinders a more pronounced reduction of the interfacial tension was observed with increasing cylinder length. ${ }^{20}$ Additional studies have suggested that parameters such as size, aspect ratio, form and shape of Janus particles play a significant role in their surface activity, particle orientation and packing geometry. ${ }^{19,21-23}$ With a view to industrial application, amphiphilic spherical Janus micelles were successfully used as stabilizers in emulsion polymerization revealing advantageous properties, including the stabilization of surface areas significantly higher compared to the cross-section of the micelles. $^{24,25}$

Patchy particles - bearing multiple different corona compartments - have only been explored to a much lower extent, ${ }^{1,26,27}$ and, in addition, mostly spherical particles were investigated. ${ }^{28-30}$ Liu et al. prepared patchy cylinders, vesicles and tubes from a triblock terpolymer via dialysis into different nonsolvents for the middle block. ${ }^{31}$ Even the formation of double and triple helices via wrapping and fusing of small patchy cylindrical micelles was observed using a similar block terpolymer. ${ }^{32}$ Lately, crystallization-driven self-assembly - known to promote the formation of one-dimensional micelles ${ }^{33-35}$ - was used to prepare worm-like micelles with a patch-like microphase separation in the corona. $^{36-38}$ It is noted that crystallization-induced selfassembly also allows for the production of crystalline-core micelles with a block-type compartmentalized corona. ${ }^{39-41}$ Although patchy particles do not share the characteristic 
geometry of Janus particles, the different hemishells on opposing sides, they should also benefit from their non-homogeneous corona in terms of surface activity. Despite the envisaged application of patchy particles for the stabilization of emulsions, ${ }^{42}$ to the best of our knowledge, the interfacial activity of particles with a patchy corona consisting of multiple compartments has not yet been investigated. Besides, there are reports on the interfacial behaviour of mixed polymer brush-grafted particles, which are able to adapt to an interface via chain reorganization. ${ }^{\mathbf{4 3 , 4 4}}$

Hence, we hereby present a study on the interfacial activity of worm-like crystalline-core micelles (wCCMs) with a patchy corona consisting of multiple polystyrene and poly(methyl methacrylate) compartments and a semi-crystalline polyethylene (PE) core. These structures significantly decrease the interfacial tension at the toluene-water interface as shown by pendant-drop tensiometry and the effect is similar to that observed for Janus cylinders of similar size. Furthermore, the influence of the corona size and composition of the patchy wCCMs on the interfacial activity is investigated.

The patchy wCCMs were self-assembled from polystyreneblock-polyethylene-block-poly(methyl methacrylate) (PS- $b$-PE- $b$ PMMA) triblock terpolymers directly in solution. The triblock terpolymers were first dissolved in toluene $\left(1 \mathrm{~g} \mathrm{~L}^{-1}\right)$ above the melting temperature of the PE middle block. Subsequently, the solution was kept at the desired temperature of isothermal crystallization $\left(T_{\mathrm{c}}\right)$ for one day to trigger crystallization-induced self-assembly into worm-like micelles with a semicrystalline PE core and a patch-like compartmentalized corona of PS and PMMA. An additional annealing step was performed to achieve a perfection of the core structure as well as a more pronounced microphase separation in the corona..$^{37,38}$ The used triblock coand terpolymers as well as the applied crystallization and annealing temperatures are summarized in Table 1. For wCCMs prepared in toluene the degree of crystallinity of the PE core is typically about $40-50 \% .{ }^{37}$ A detailed morphological characterization of the investigated wCCMs can be found in our previous publications. ${ }^{37,38}$ Selected TEM micrographs of SEM and SES wCCMs (Fig. $\mathrm{S} 1 \dagger$ ) as well as experimental details are provided in the ESI. $\dagger$
For this study, the toluene-water interface was chosen for mainly two reasons. Firstly, the wCCMs can directly be selfassembled in toluene and, secondly, toluene and water are immiscible and exhibit a high interfacial tension, criteria that are essential to achieve reproducible results. In these measurements toluene was used as the solution phase and water, due to its higher density, as the droplet phase. The interfacial tension at the pristine toluene-water interface was determined to be $\gamma_{0}=33.1 \mathrm{mN} \mathrm{m}^{-1}$ (Fig. $\mathrm{S} 2 \dagger$ ).

To clarify whether the combination of the "Pickering effect" of the worm-like micelles with slightly amphiphilic properties due to the patchy PS/PMMA corona results in a synergistic effect, wCCMs formed by a symmetric PS- $b$-PE- $b$-PMMA triblock terpolymer (SEM1, equal length of end blocks) were compared to PS- $b$-PB- $b$-PMMA (SBM, PB = polybutadiene) unimers and wCCMs with a homogeneous PS corona formed by a PS- $b$-PE- $b$ PS (SES) triblock copolymer. A schematic depiction of the investigated one-dimensional micellar structures can be found in Scheme 1. The used SBM is the non-hydrogenated PB-containing precursor triblock terpolymer of SEM1 and, thus, the lengths of the PS and PMMA blocks are identical. However, as the PB block is amorphous and well soluble in toluene at ambient temperature, in contrast to the PE block in SEM1, the SBM triblock terpolymer is molecularly dissolved. The wormlike SES micelles with a homogeneous PS corona exhibit a comparable length with respect to that of the SEM1 wCCMs and bear corona blocks with a similar degree of polymerization (Table 1). Thus, the interfacial activities of SBM unimers and SES wCCMs represent suitable benchmarks for SEM1 wCCMs.

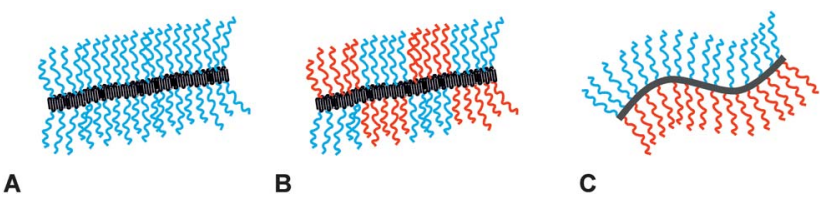

Scheme 1 Overview of the investigated micelles: wCCMs with (A) homogeneous and ( $B$ ) patchy corona, and (C) Janus cylinders (PS = blue, PMMA = red, core $=$ semi-crystalline $\mathrm{PE}(\mathrm{A}$ and $\mathrm{B})$ or crosslinked $\mathrm{PB}(\mathrm{C}))$.

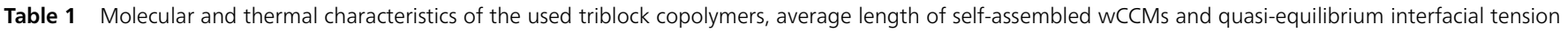

\begin{tabular}{|c|c|c|c|c|c|c|}
\hline Sample & Polymer $^{a}$ & $\mathrm{DP}_{\text {corona }} / x_{\mathrm{PS}}^{b}$ & $T_{\mathrm{c}}{ }^{c}\left[{ }^{\circ} \mathrm{C}\right]$ & $T_{\mathrm{a}}{ }^{c}\left[{ }^{\circ} \mathrm{C}\right]$ & $l^{d}[\mathrm{~nm}]$ & $\gamma^{e}\left[\mathrm{mN} \mathrm{m}^{-1}\right]$ \\
\hline SEM1 & $\mathrm{S}_{340} \mathrm{E}_{700} \mathrm{M}_{360}$ & $700 / 0.49$ & 20 & 45 & $520(140)$ & $17.6(0.3)$ \\
\hline SEM2 & $\mathrm{S}_{140} \mathrm{E}_{690} \mathrm{M}_{160}$ & $300 / 0.47$ & 30 & 40 & $560(130)$ & $19.4(0.1)$ \\
\hline SEM3 & $\mathrm{S}_{280} \mathrm{E}_{1190} \mathrm{M}_{300}$ & $580 / 0.48$ & 34 & 48 & $540(160)$ & $18.0(0.1)$ \\
\hline SEM4 & $\mathrm{S}_{330} \mathrm{E}_{1360} \mathrm{M}_{760}$ & $1090 / 0.3$ & 20 & 40 & $780(240)$ & $18.1(0.1)$ \\
\hline SEM5 & $\mathrm{S}_{490} \mathrm{E}_{1470} \mathrm{M}_{610}$ & $1100 / 0.45$ & 20 & 40 & $850(250)$ & $17.7(0.2)$ \\
\hline SEM6 & $\mathrm{S}_{660} \mathrm{E}_{1350} \mathrm{M}_{350}$ & $1010 / 0.65$ & 18 & 40 & $710(200)$ & $18.9(0.1)$ \\
\hline SES & $S_{380} E_{880} S_{390}$ & $770 / 1$ & 20 & 45 & $690(230)$ & $19.4(0.1)$ \\
\hline Janus $^{20}$ & $\mathrm{~S}_{430} \mathrm{~B}_{290} \mathrm{M}_{500}$ & $930 / 0.46$ & - & - & $800(250)$ & $18.0(0.1)$ \\
\hline $\operatorname{SBM}^{f}$ & $\mathrm{~S}_{340} \mathrm{~B}_{350} \mathrm{M}_{360}$ & $700 / 0.49$ & - & - & - & $19.4(0.4)$ \\
\hline
\end{tabular}

${ }^{a}$ Subscripts give the number-average degree of polymerization of the respective block. ${ }^{b}$ Overall degree of polymerization of the corona blocks PS and PMMA (DP corona $)$ and molar fraction of PS units in the wCCM corona $\left(x_{\mathrm{PS}}\right) .{ }^{c}$ Applied temperatures of isothermal crystallization $\left(T_{\mathrm{c}}\right)$ and annealing $\left(T_{\mathrm{a}}\right)$, respectively. ${ }^{d}$ Average wCCM length derived from TEM image analysis (standard deviation in parentheses). ${ }^{e}$ Quasi-equilibrium interfacial tension determined by averaging the values of the interfacial tension measured during the last 30 min of the experiment (standard deviation in parentheses). ${ }^{f}$ Precursor of SEM1 prior to hydrogenation, unimolecularly dissolved in toluene. 
The corresponding pendant drop tensiometer measurements are displayed in Fig. 1A. The interfacial tension at the water-toluene interface shows a rapid decrease at early stages of adsorption for all samples, which levels off and finally reaches a plateau (quasi-equilibrium interfacial tension). In the presence of SEM1 wCCMs the quasi-equilibrium interfacial tension $\left(\gamma(\mathrm{SEM} 1)=17.6 \mathrm{mN} \mathrm{m}^{-1}\right)$ is significantly lower than for SBM unimers $\left(\gamma(\mathrm{SBM})=19.4 \mathrm{mN} \mathrm{m}^{-1}\right)$ and SES wCCMs $(\gamma(\mathrm{SES})=$ $19.4 \mathrm{mN} \mathrm{m}^{-1}$ ), highlighting the beneficial combination of surface compartmentalization (amphiphilicity) with the "Pickering effect" of the wCCMs. The considerable reduction of the interfacial tension even in the presence of SBM unimers is ascribed to the difference in the PS-water and PMMA-water interfacial tensions of 32 and $16 \mathrm{mN} \mathrm{m}^{-1}$, respectively. ${ }^{19}$ Accordingly, PMMA has a significantly higher affinity to the water phase compared to PS, irrespective of the fact that PMMA is not soluble in water. While for SBM unimers the interfacial tension reaches a constant value at relatively short times, for solutions containing SES and SEM1 wCCMs establishing a quasi-equilibrium state takes about $2 \mathrm{~h}$. On one hand, the diffusion of unimers to the droplet surface might be faster than for considerably larger micellar structures and, on the other hand, the arrangement of the wCCMs at the interface is
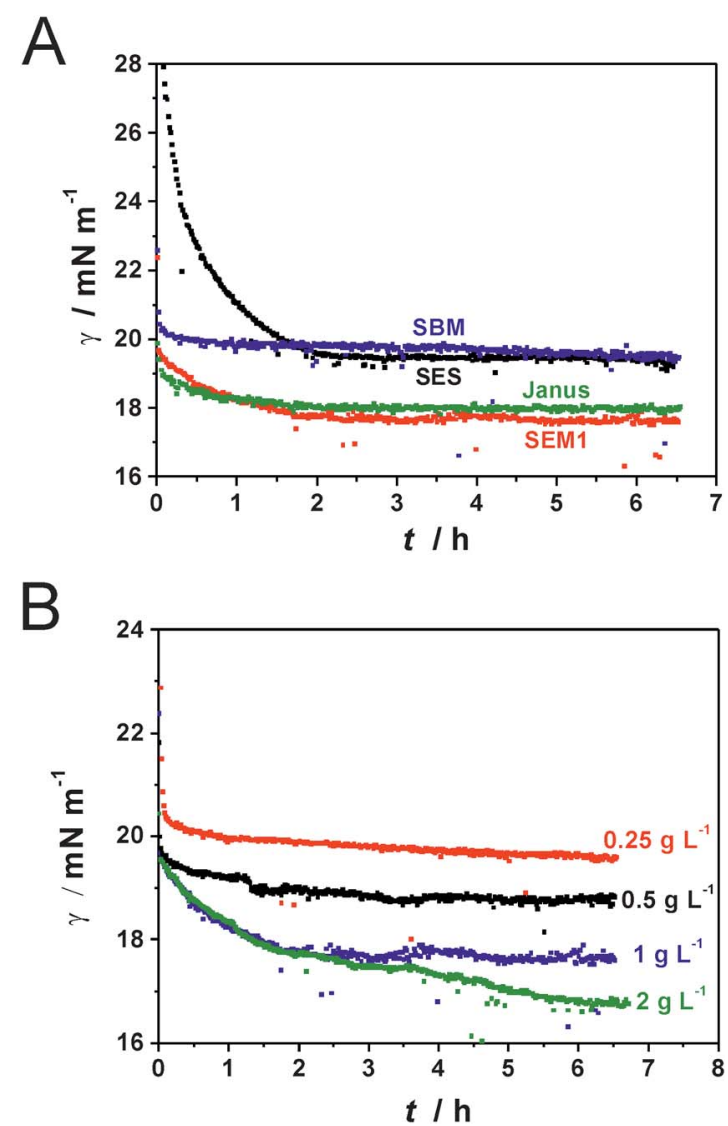

Fig. 1 (A) Interfacial tension isotherms of $1 \mathrm{~g} \mathrm{~L}^{-1}$ solutions containing SBM unimers, SES wCCMs with a homogeneous corona, SEM1 wCCMs with a patchy corona and SBM Janus cylinders. (B) Interfacial tension isotherms of differently concentrated solutions of wCCMs formed by SEM1. supposed to take a much longer time. Moreover, the interfacial tension for SES wCCMs decreases rather slowly, whereas SEM1 reaches values below $20 \mathrm{mN} \mathrm{m}^{-1}$ much faster, pointing to a higher affinity of the patchy wCCMs toward the interface attributable to the weak amphiphilic character of the patchy corona. In the logarithmic presentation of the data for SEM1 wCCMs, different stages of adsorption appear more precisely (Fig. S3 $\dagger$ ). In the first stage the interfacial tension decreases rapidly due to free diffusion of the wCCMs to the interface. Subsequently, the decrease slows down (stage II) going along with ordering and domain formation at the interface. Finally, the interfacial tension approaches a plateau indicating the maximum coverage of the interface (stage III). In addition, we studied the concentration dependence of the interfacial tension and found a significant decrease of the quasi-equilibrium interfacial tension upon increasing concentration of SEM1 based wCCMs in the toluene phase (Fig. 1B). A comparable behaviour was observed for SBM Janus cylinders, too, and is attributed to an increasing surface pressure of adsorbing wCCMs with increasing concentration. ${ }^{20}$

The interfacial activity of SEM1 wCCMs at the toluene-water interface was directly compared to that of SBM Janus cylinders used in a previous study. ${ }^{20}$ Strikingly, the quasi-equilibrium interfacial tension in the presence of the Janus cylinders $\left(\gamma(\right.$ Janus $\left.)=18.0 \mathrm{mN} \mathrm{m}^{-1}\right)$ is within the accuracy of the technique in the same range as for SEM1 (Fig. 1A). Here, it has to be noted that the length of the PS and PMMA corona blocks and the total length of the Janus micelles are higher than those of the wCCMs formed by SEM1, while the molar fraction of PS units in the corona is comparable for both (Table 1). Consequently, a higher reduction of the interfacial tension in the case of the Janus cylinders might be expected as an increase in the average length of Janus cylinders was shown to enhance the adsorption at the interface and, thus, results in lower plateau values. ${ }^{20}$ In order to examine whether this unexpected finding occurs only for the system toluene-water, we studied the interfacial activity of SEM1 wCCMs with dioxane as the solution phase and perfluorooctane (PFO) as the droplet phase, too. Here, prior investigations resulted in a quasi-equilibrium interfacial tension of $\gamma(\mathrm{Janus})=7.7 \mathrm{mN} \mathrm{m}^{-1}$ in the presence of Janus cylinders with a length of $800 \mathrm{~nm},{ }^{20}$ and again the plateau value of the patchy wCCMs was found to be comparable $\left(\gamma(\right.$ SEM1 $)=7.3 \mathrm{mN} \mathrm{m}^{-1}$, Fig. S4 $\left.\dagger\right)$.

In the first place, one would assume that Janus cylinders exhibit a higher interfacial activity compared to cylindrical structures with a patchy corona. The segregation of the PS/PMMA corona into two hemishells should be ideally suited to arrange at the liquid-liquid interface. However, wCCMs with multiple compartments of PS and PMMA obviously achieve similar results. This leads to the assumption that the corona chains are able to adapt to the interface. Based on simulations and experiments on PS/PMMA Janus cylinders, which showed a preferential orientation of the PS chains to the toluene phase and the PMMA chains to the water phase, ${ }^{19}$ the following model for patchy wCCMs is proposed (Scheme 2). In the toluene phase PS and PMMA are soluble, whereas PS exhibits a slightly better solubility compared to PMMA. ${ }^{45}$ Although water is a non-solvent 


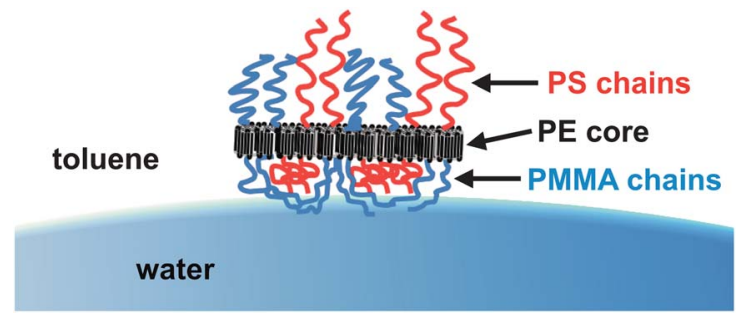

Scheme 2 Proposed orientation of patchy wCCMs at the toluene-water interface and adaptation of the corona chains.

for both corona blocks, the more polar PMMA chains are most likely oriented toward the water phase and probably act as a shielding layer for the even less polar PS blocks and the PE core. Conversely, the PS chains at the same side of the wCCMs facing the water droplet surface collapse close to the PE core or even fold around the core in order to reach into the toluene phase where possible.

Prior studies on Janus cylinders already revealed a strong influence of the cylinder length on the interfacial properties. ${ }^{20}$ However, the impact of the corona composition and the corona thickness has not yet been addressed. In order to investigate the influences of the corona structure in more detail, the toluenewater interfacial tension in the presence of two series of SEM
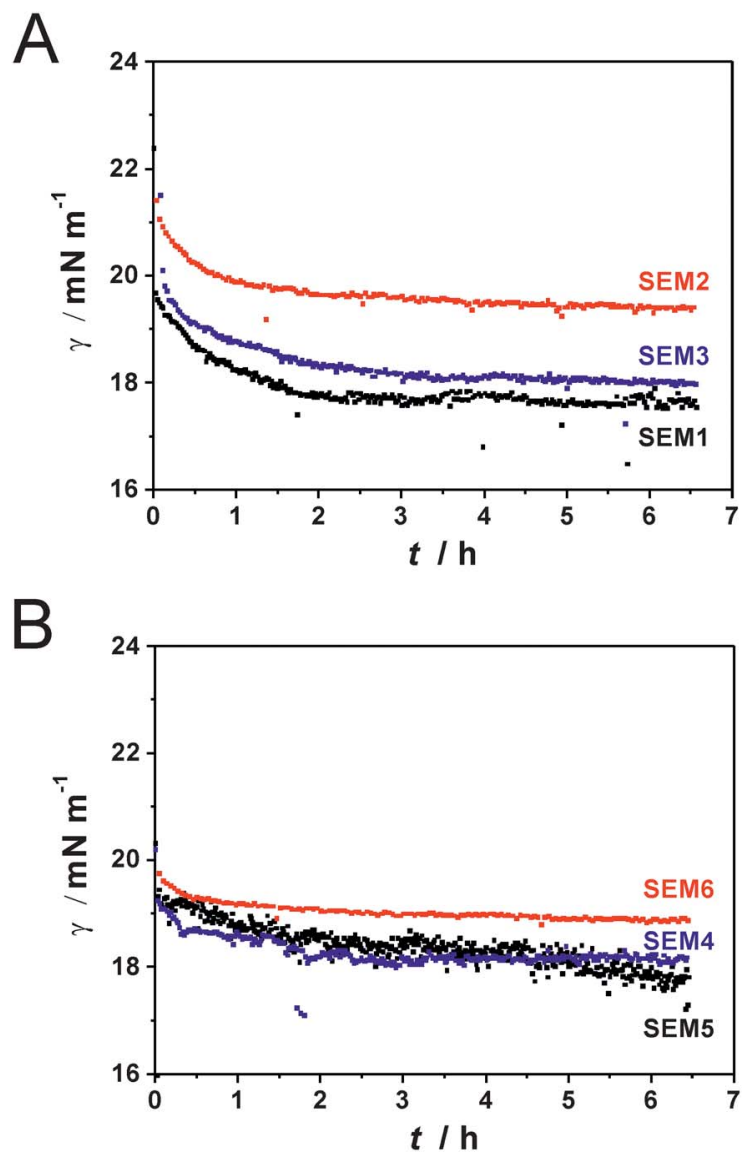

Fig. 2 Interfacial tension isotherms of $1 \mathrm{~g} \mathrm{~L}^{-1}$ solutions containing wCCMs formed by SEM1-3 (A) and SEM4-6 (B). based wCCMs was studied. Notably, the average length of the formed wCCMs in each series did not vary significantly (Table 1) and, thus, is not expected to exert a significant influence on the interfacial activity. In the first series, SEM1-3, the molar fraction of PS units $\left(x_{\mathrm{PS}}\right)$ in the corona of the wCCMs was kept constant, while the overall degree of polymerization ( $\mathrm{DP}_{\text {corona }}$ ) of both corona blocks was varied. The interfacial tension isotherms displayed in Fig. 2A clearly show an increase in interfacial activity, i.e., decrease in interfacial tension, upon increasing length of the corona blocks. The quasi-equilibrium interfacial tension decreases continuously from $\gamma(\operatorname{SEM} 2)=19.4 \mathrm{mN} \mathrm{m}^{-1}$ for $\mathrm{DP}_{\text {corona }}=300$ down to $\gamma(\mathrm{SEM} 1)=17.6 \mathrm{mN} \mathrm{m}^{-1}$ for $\mathrm{DP}_{\text {corona }}$ $=700$. This shows that a more extended corona of the worm-like micelles enhances their ability to stabilize interfaces, which is in agreement with theoretical predictions. ${ }^{6}$ In the second series, SEM4-6, wCCMs with comparable overall degree of polymerization of the corona blocks but different molar fractions of PS units in the corona were examined (Fig. 2B). Here, the interfacial tension isotherms exhibit only a weak dependence on the corona composition, with quasi-equilibrium interfacial tensions for wCCMs with an asymmetric corona composition $\left(\gamma(\right.$ SEM4 $)=18.1 \mathrm{mN} \mathrm{m}^{-1}$ and $\gamma\left(\right.$ SEM6) $\left.=18.9 \mathrm{mN} \mathrm{m}^{-1}\right)$ being slightly higher compared to wCCMs with a symmetric corona composition $\left(\gamma(\right.$ SEM5 $\left.)=17.7 \mathrm{mN} \mathrm{m}^{-1}\right)$. This points to a minor influence of the corona composition on the interfacial properties of patchy wCCMs within the investigated range.

\section{Conclusions}

A first study of the interfacial activity of self-assembled wormlike crystalline-core micelles (wCCMs) with polyethylene (PE) cores and a patchy corona of polystyrene (PS) and poly(methyl methacrylate) (PMMA) at the toluene-water interface is presented. The ability of these patch-like surface-compartmentalized nanostructures to reduce the quasi-equilibrium interfacial tension is significantly higher compared to that of single chains of the precursor triblock terpolymer or wCCMs with a homogeneous PS corona. This highlights the beneficial synergy of the particular nature of the investigated wCCMs ("Pickering effect") and the amphiphilicity of the patchy PS/PMMA corona. Strikingly, the interfacial activity of the investigated patchy wCCMs is comparable to that of SBM Janus cylinders with similar dimensions and corona composition, even though the Janus structure with two corona hemishells of PS and PMMA might be regarded as more suitable for the stabilization of interfaces than micelles with multiple corona patches on both sides. Screening of a series of wCCMs formed by different PS- $b$-PE- $b$-PMMA triblock terpolymers with varying compositions and molecular weights revealed that the overall degree of polymerization of the corona chains, i.e., the thickness of the corona, is a crucial parameter that influences the interfacial activity of these nanostructures. Besides, the composition of the corona in terms of the molar fraction of PS units seems to play a minor role. From the presented results a rearrangement of the wCCM corona chains at the liquid-liquid interface is proposed, rendering patchy wCCMs a new class of adaptive surfactants. Due to the comparably simple preparation of these structures 
directly in solution by crystallization-induced self-assembly, applications in the stabilization of emulsions and/or polymer blends can be imagined. Future investigation will concentrate on the production of wCCMs with a strong amphiphilic corona by incorporating water-soluble polymer blocks.

\section{Acknowledgements}

This work was funded by the German Science Foundation via the collaborative research centre SFB 840 (project A2). J.S. thanks the Elite Network of Bavaria for support.

\section{Notes and references}

1 J. Du and R. K. O'Reilly, Chem. Soc. Rev., 2011, 40, 2402-2416.

2 K. Zhang, M. Jiang and D. Chen, Prog. Polym. Sci., 2012, 37, 445-486.

3 S. U. Pickering, J. Chem. Soc. Trans., 1907, 91, 2001-2021.

4 W. Ramsden, Proc. R. Soc. London, 1903, 72, 156-164.

5 P. Pieranski, Phys. Rev. Lett., 1980, 45, 569-572.

6 A. Böker, J. He, T. Emrick and T. P. Russell, Soft Matter, 2007, 3, 1231-1248.

7 J. Hu, S. Zhou, Y. Sun, X. Fang and L. Wu, Chem. Soc. Rev., 2012, 41, 4356-4378.

8 A. Walther and A. H. E. Müller, Chem. Rev., 2013, 113, 51945261.

9 V. N. Paunov and O. J. Cayre, Adv. Mater., 2004, 16, 788-791.

10 L. Hong, S. Jiang and S. Granick, Langmuir, 2006, 22, 94959499.

11 K.-H. Roh, D. C. Martin and J. Lahann, Nat. Mater., 2005, 4, 759-763.

12 Z. Nie, W. Li, M. Seo, S. Xu and E. Kumacheva, J. Am. Chem. Soc., 2006, 128, 9408-9412.

13 R. Erhardt, A. Böker, H. Zettl, H. Kaya, W. Pyckhout-Hintzen, G. Krausch, V. Abetz and A. H. E. Müller, Macromolecules, 2001, 34, 1069-1075.

14 Y. Liu, V. Abetz and A. H. E. Müller, Macromolecules, 2003, 36, 7894-7898.

15 A. Wolf, A. Walther and A. H. E. Müller, Macromolecules, 2011, 44, 9221-9229.

16 A. Walther, X. André, M. Drechsler, V. Abetz and A. H. E. Müller, J. Am. Chem. Soc., 2007, 129, 6187-6198.

17 B. P. Binks and P. D. I. Fletcher, Langmuir, 2001, 17, 47084710.

18 N. Glaser, D. J. Adams, A. Böker and G. Krausch, Langmuir, 2006, 22, 5227-5229.

19 T. M. Ruhland, A. H. Gröschel, N. Ballard, T. S. Skelhon, A. Walther, A. H. E. Müller and S. A. F. Bon, Langmuir, 2013, 29, 1388-1394.
20 T. M. Ruhland, A. H. Gröschel, A. Walther and A. H. E. Müller, Langmuir, 2011, 27, 9807-9814.

21 B. J. Park, T. Brugarolas and D. Lee, Soft Matter, 2011, 7, 6413-6417.

22 B. J. Park and D. Lee, ACS Nano, 2011, 6, 782-790.

23 B. J. Park and D. Lee, Soft Matter, 2012, 8, 7690-7698.

24 A. Walther, M. Hoffmann and A. H. E. Müller, Angew. Chem., Int. Ed., 2008, 47, 711-714.

25 R. Ayeward, Soft Matter, 2012, 8, 5233-5240.

26 A. B. Pawar and I. Kretzschmar, Macromol. Rapid Commun., 2010, 31, 150-168.

27 J. Yoon, K. J. Lee and J. Lahann, J. Mater. Chem., 2011, 21, 8502-8510.

28 D. A. Christian, A. Tian, W. G. Ellenbroek, I. Levental, K. Rajagopal, P. A. Janmey, A. J. Liu, T. Baumgart and D. E. Discher, Nat. Mater., 2009, 8, 843-849.

29 S. C. Glotzer and M. J. Solomon, Nat. Mater., 2007, 6, 557562.

30 M. D. McConnell, M. J. Kraeutler, S. Yang and R. J. Composto, Nano Lett., 2010, 10, 603-609.

31 G. Njikang, D. H. Han, J. Wang and G. J. Liu, Macromolecules, 2008, 41, 9727-9735.

32 J. Dupont, G. J. Liu, K. Niihara, R. Kimoto and H. Jinnai, Angew. Chem., Int. Ed., 2009, 48, 6144-6147.

33 M. Lazzari and M. A. Lopez-Quintela, Macromol. Rapid Commun., 2009, 30, 1785-1791.

34 J. B. Gilroy, T. Gädt, G. R. Whittell, L. Chabanne, J. M. Mitchels, R. M. Richardson, M. A. Winnik and I. Manners, Nat. Chem., 2010, 2, 566-570.

35 J. Schmelz, F. H. Schacher and H. Schmalz, Soft Matter, 2013, 9, 2101-2107.

36 H. Schmalz, J. Schmelz, M. Drechsler, J. Yuan, A. Walther, K. Schweimer and A. M. Mihut, Macromolecules, 2008, 41, 3235-3242.

37 J. Schmelz, M. Karg, T. Hellweg and H. Schmalz, ACS Nano, 2011, 5, 9523-9534.

38 J. Schmelz and H. Schmalz, Polymer, 2012, 53, 4333-4337.

39 T. Gädt, N. S. Ieong, G. Cambridge, M. A. Winnik and I. Manners, Nat. Mater., 2009, 8, 144-150.

40 F. He, T. Gädt, I. Manners and M. A. Winnik, J. Am. Chem. Soc., 2011, 133, 9095-9103.

41 P. A. Rupar, L. Chabanne, M. A. Winnik and I. Manners, Science, 2012, 337, 559-562.

42 S. Granick and L. Hong, US Pat., 0305219 A1, 2010.

43 D. Priftis, G. Sakellariou, D. Baskaran, J. W. Mays and N. Hadjichristidis, Soft Matter, 2009, 5, 4272-4278.

44 B. Zhao and L. Zhu, Macromolecules, 2009, 42, 9369-9383. 45 M. S. Kent, M. Tirrell and T. P. Lodge, J. Polym. Sci., Part B: Polym. Phys., 1994, 32, 1927-1941. 